\title{
Continuum-wise expansiveness for generic diffeomorphisms
}

\author{
Manseob Lee \\ Department of Mathematics, Mokwon University, \\ Daejeon, 302-729, Korea. \\ E-mail:lmsds@mokwon.ac.kr.
}

\begin{abstract}
Let $M$ be a closed smooth manifold and let $f: M \rightarrow M$ be a diffeomorphism. $C^{1}$-generically, a continuum-wise expansive satisfies Axiom A without cycles. Let $M=\mathbb{T}^{3}$ and let $f: \mathbb{T}^{3} \rightarrow \mathbb{T}^{3}$. There are a $C^{1}$ neighborhood $\mathcal{U}(f)$ of $f \in \mathcal{R} \mathcal{T}\left(\mathbb{T}^{3}\right)$ and a residual set $\mathcal{R} \subset \mathcal{U}(f)$ such that for any $g \in \mathcal{R}, g$ is not continuum-wise expansive, where $\mathcal{R} \mathcal{T}\left(\mathbb{T}^{3}\right)$ is the set of all robustly transitive diffeomorphisms on $\mathbb{T}^{3}$.
\end{abstract}

\section{Introduction}

Let $M$ be a closed smooth manifold with $\operatorname{dim} M \geq 2$, and let $\operatorname{Diff}(M)$ be the space of diffeomorphisms of $M$ endowed with the $C^{1}$ topology. Denote by $d$ the distance on $M$ induced from a Riemannian metric $\|\cdot\|$ on the tangent bundle $T M$. In differentiable dynamical systems, expansiveness is a very useful notion to investigate for stability theory. For instance, Mañé [16] proved that the $C^{1}$-interior of the set of expansive diffeomorphisms coincides with the set of quasi-Anosov diffeomorphisms. Here $f$ is quasi-Anosov if for all $v \in T M \backslash\{0\}$, the set $\left\{\left\|D f^{n}(v)\right\|: n \in \mathbb{Z}\right\}$ is unbounded. Let $f \in \operatorname{Diff}(M)$. We say that $f$ is expansive if there is $e>0$ such that for any $x, y \in M$ if $d\left(f^{i}(x), f^{i}(y)\right)<e$ for all $i \in \mathbb{Z}$ then $x=y$. Denote by $\mathcal{E}$ the set of all expansive diffeomorphisms. From now, we introduce various expansiveness (N-expansive, countably expansive, measure expansive [18, 19]) which are general notions of original expansiveness.

\footnotetext{
${ }^{1} 2010$ Mathematical Subject Classification: 37D30; 34D10.

Key words and phrases: expansive; continuum-wise expansive; Axiom A; partially hyperbolic; generic.
} 
We say that $f$ is $N$-expansive if there is $e>0$ such that for any $x \in M$, the number of elements of the set $\Gamma_{e}(x)=\left\{y \in M: d\left(f^{i}(x), f^{i}(y)\right)<e\right.$ for all $\left.i \in \mathbb{Z}\right\}$ is less than $N$. Denote by $\mathcal{G} \mathcal{E}$ the set of all $\mathrm{N}$-expansive diffeomorphisms on $M$. We say that $f$ is countably expansive if there is $e>0$ such that for $x \in M$, the number of elements of the set $\Gamma_{e}(x)=\left\{y \in M: d\left(f^{i}(x), f^{i}(y)\right)<e\right.$ for all $\left.i \in \mathbb{Z}\right\}$ is countable, where $e$ is an expansive constant for $f$.

Note that if a diffeomorphism $f$ is expansive then $\Gamma_{e}(x)=\{x\}$ for $x \in M$. Thus if a diffeomorphism $f$ is expansive then $f$ is countably expansive, but the converse is not true (see [19]).

For a Borel probability measure $\mu$ on $M$, we say that $f$ is $\mu$-expansive if there is $\delta>0$ such that $\mu\left(\Gamma_{e}(x)\right)=0$ for all $x \in M$. In this case, we say that $\mu$ is a expansive measure for $f$. We say that $f$ is measure expansive if it is $\mu$-expansive for every non-atomic Borel probability measure $\mu$. Denote by $\mathcal{M E}$ the set of all measure-expansive diffeomorphisms on $M$.

Continuum-wise expansive diffeomorphisms was introduced by Kato [12]. A set $\Lambda$ is nondegenerate if the set $\Lambda$ is not reduced to one point. We say that $\Lambda \subset M$ is a subcontinuum if it is a compact connected nondegenerate subset of $M$.

Definition 1.1 A diffeomorphism $f$ on $M$ is said to be continuum-wise expansive if there is a constant $e>0$ such that for any nondegenerate subcontinuum $\Lambda$ there is an integer $n=n(\Lambda)$ such that $\operatorname{diam} f^{n}(\Lambda) \geq e$, where $\operatorname{diam} \Lambda=\sup \{d(x, y): x, y \in \Lambda\}$ for any subset $\Lambda \subset M$. Such a constant $e$ is called a continuum-wise expansive constant for $f$.

Note that every expansive diffeomorphism is continuum-wise expansive diffeomorphism, but its converse is not true (see [12, Example 3.5]). Denote by $\mathcal{C} \mathcal{W} \mathcal{E}$ the set of all continuum-wise expansive diffeomorphisms of $M$. In [4], Artigue showed that

$$
\mathcal{E} \Rightarrow \mathcal{G E} \Rightarrow \mathcal{C E}=\mathcal{M E} \Rightarrow \mathcal{C W E}
$$

where $\mathcal{C} \mathcal{E}$ is the set of all countably expansive diffeomorphisms on $M$. For a $C^{1}$ perturbation expansive diffeomorphism, we can find the following result (see [5, 13, 21, 22]). Denote by int $A$ the $C^{1}$-interior of a set $A$ of $C^{1}$-diffeomorphisms of $M$.

Theorem 1.2 Let $f \in \operatorname{Diff}(M)$. Then we have the following

$$
\text { int } \mathcal{E}=i n t \mathcal{G E}=\operatorname{int} \mathcal{M E}=\operatorname{int} \mathcal{C} \mathcal{W} \mathcal{E}
$$

Let $\Lambda$ be a closed $f$-invariant set. We say that $\Lambda$ is hyperbolic if the tangent bundle $T_{\Lambda} M$ has a $D f$-invariant splitting $E^{s} \oplus E^{u}$ and there exists constants $C>0$ and $0<\lambda<1$ such that

$$
\left\|\left.D_{x} f^{n}\right|_{E_{x}^{s}}\right\| \leq C \lambda^{n} \text { and }\left\|\left.D_{x} f^{-n}\right|_{E_{x}^{u}}\right\| \leq C \lambda^{n}
$$


for all $x \in \Lambda$ and $n \geq 0$. If $\Lambda=M$ then $f$ is said to be Anosov.

It is well know that if a diffeomorphism $f$ is Anosov then it is quasi-Anosov, but the converse is not true (see [10]). Thus if a diffeomorphism $f$ is Anosov then $f$ is expansive, N-expansive, measure expansive, countably expansive and continuum-wise expansive. We say that $f$ satisfies Axiom $A$ if the non-wandering set $\Omega(f)$ is hyperbolic and it is the closure of $P(f)$. A point $x \in M$ is said to be non-wandering for $f$ if for any non-empty open set $U$ of $x$ there is $n \geq 0$ such that $f^{n}(U) \cap U \neq \emptyset$. Denote by $\Omega(f)$ the set of all non-wandering points of $f$. It is clear that $\overline{P(f)} \subset \Omega(f)$. A diffeomorphism $f$ is $\Omega$-stable if there is a $C^{1}$-neighborhood $\mathcal{U}(f)$ of $f$ such that for any $g \in \mathcal{U}(f)$ there is a homeomorphism $h: \Omega(f) \rightarrow \Omega(g)$ such that $h \circ f=g \circ h$, where $\Omega(g)$ is the non-wandering set of $g$. A subset $\mathcal{G} \subset \operatorname{Diff}(M)$ is called residual if it contains a countable intersection of open and dense subsets of $\operatorname{Diff}(M)$. A dynamic property is called $C^{1}$ generic if it holds in a residual subset of $\operatorname{Diff}(M)$. Arbieto [3] proved that if a $C^{1}$ generic diffeomorphism $f$ is expansive then it is Axiom A without cycles. Lee [13] proved that if a $C^{1}$ generic diffeomorphism $f$ is $\mathrm{N}$-expansive then it is Axiom A without cycles. Very recently, Lee [15] proved that if a $C^{1}$ generic diffeomorphism $f$ is measure expansive then it is Axiom A without cycles. From that, we consider $C^{1}$ generic continuum-wise expansive diffeomorphisms. The following is a main result.

Theorem A For $C^{1}$ generic $f \in \operatorname{Diff}(M)$, if $f$ is continuum-wise expansive then it is Axiom A without cycles.

We say that a $f$-invariant closed set $\Lambda$ admits a dominated splitting if the tangent bundle $T_{\Lambda} M$ has a continuous $D f$-invariant splitting $E \oplus F$ and there exist constants $C>0$ and $0<\lambda<1$ such that

$$
\left\|\left.D_{x} f^{n}\right|_{E(x)}\right\| \cdot\left\|\left.D_{x} f^{-n}\right|_{F\left(f^{n}(x)\right)}\right\| \leq C \lambda^{n}
$$

for all $x \in \Lambda$ and $n \geq 0$. If the dominated splitting can be written as a sum

$$
T_{\Lambda} M=E_{1} \oplus E_{2} \oplus \cdots \oplus E_{i} \oplus E_{i+1} \oplus \cdots \oplus E_{k},
$$

then we say that the sum is dominated if for all $i$ the sum

$$
\left(E_{1} \oplus E_{2} \oplus \cdots \oplus E_{i}\right) \oplus\left(E_{i+1} \oplus E_{i+2} \oplus \cdots E_{k}\right)
$$

is dominated. Note that the decomposition is called the finest dominated splitting if we can't decompose in a non-trivial way subbundle $E_{i}$ appearing in the splitting.

The set $\Lambda$ is partially hyperbolic if there is a dominated splitting $E \oplus F$ of $T_{\Lambda} M$ such that either $E$ is contracting or $F$ is expanding. 
Definition 1.3 We say that a compact $f$-invariant set $\Lambda \subset M$ is strongly partially hyperbolic if the tangent bundle $T_{\Lambda} M$ has a dominated splitting $E^{s} \oplus E^{c} \oplus E^{u}$ and there exist $C>0$ and $0<\lambda<1$ such that for all $v \in E^{s}$, we have $\left\|D f^{n}(v)\right\| \leq C \lambda^{n}\|v\|$ for all $n \geq 0$, and for all $v \in E^{u}$, we have $\left\|D f^{-n}(v)\right\| \leq C \lambda^{n}\|v\|$ for all $n \geq 0$, where $E^{c}$ is the central direction of the splitting.

Note that if $\Lambda$ is hyperbolic for $f$ then it is strongly partially hyperbolic and $E^{c}$ is not empty, that is, $E^{c}=\{0\}$. For a partially hyperbolic diffeomorphism, Burns and Wilkinson [6] showed the following lemma.

Lemma 1.4 Let $\Lambda$ be a compact $f$-invariant set with a partially hyperbolic splitting,

$$
T_{\Lambda} M=E^{s} \oplus E_{1}^{c} \oplus \cdots \oplus E_{k}^{c} \oplus E^{u} .
$$

Let $E^{c s, i}=E^{s} \oplus E_{1}^{c} \oplus \cdots \oplus E_{i}^{c}$ and $E^{c u, i}=E_{i}^{c} \oplus \cdots \oplus E_{k}^{c} \oplus E^{u}$ and consider their extensions $\widetilde{E}^{c s, i}$ and $\widetilde{E}^{c u, i}$ to a small neighborhood of $\Lambda$. Then for any $\epsilon>0$ there exist constants $R>r>r_{1}>0$ such that for any $x \in \Lambda$, the neighborhood $B(x, r)$ is foliated by foliations $\widehat{W}^{u}(x), \widehat{W}^{s}(x), \widehat{W}^{c s, i}(x)$ and $\widehat{W}^{c u, i}(x)(i=1, \ldots, k)$ such that for each $\sigma \in\{u, s,(c s, i),(c u, i)\}$ the following properties hold.

(a) Almost tangency of invariant distributions. For each $y \in B(x, r)$, the leaf $\widehat{W}_{x}^{\sigma}(y)$ is $C^{1}$, and the tangent space $T_{y} \widehat{W}_{x}^{\sigma}(y)$ lies in a cone of radius $\epsilon$ about $\widetilde{E}^{\sigma}(y)$.

(b) Coherence. $\widehat{W}_{x}^{s}$ subfoliates $\widehat{W}_{x}^{c s, i}$ and $\widehat{W}_{x}^{u}$ subfoliates $\widehat{W}_{x}^{c u, i}$ for each $i \in\{1, \ldots, k\}$.

(c) Local invariance. For each $y \in B(x, r)$ we have $f\left(\widehat{W}_{x}^{\sigma}\left(y, r_{1}\right)\right) \subset \widehat{W}_{f(x)}^{\sigma}(f(y))$ and $f^{-1}\left(\widehat{W}_{x}^{\sigma}\left(y, r_{1}\right)\right) \subset \widehat{W}_{f^{-1}(x)}^{\sigma}\left(f^{-1}(y)\right)$, where $\widehat{W}_{x}^{\sigma}\left(y, r_{1}\right)$ is the connected components of $\widehat{W}_{x}^{\sigma}(y) \cap B\left(y, r_{1}\right)$ containing $y$.

(d) Uniqueness. $\widehat{W}_{x}^{s}(x)=W^{s}(x, r)$ and $\widehat{W}_{x}^{u}(x)=W^{u}(x, r)$.

We say that a diffeomorphism $f$ has a homoclinic tangency if there is a hyperbolic periodic point $p$ whose invariant manifolds $W^{s}(p)$ and $W^{u}(p)$ have a non-transverse intersection. The set of $C^{1}$ diffeomorphisms that have some homoclinic tangencies will be denoted $\mathcal{H} \mathcal{T}$. For a homoclinic tangency, Pacifico and Vieitez 20] proved that surface diffeomorphisms presenting homoclinic tangencies can be $C^{1}$-approximated by non-measure expansive diffeomorphisms. Form the result, Lee 14 proved that if $f$ has a homoclinic tangency associated to a hyperbolic periodic point $p$, then there is a $g C^{1}$-close to $f$ such that $g$ is not continuum-wise expansive. 
Proposition 1.5 [7, Theorem 1.1] The diffeomorphism $f$ in a dense $\mathcal{G}_{\delta}$ subset $\mathcal{G} \subset$ $\operatorname{Diff}(M) \backslash \overline{\mathcal{H T}}$ has the following properties.

(a) Any aperiodic class $\mathcal{C}$ is partially hyperbolic with a one-dimensional central bundle. Morevoer, the Lyapunov exponent along $E^{c}$ of any invariant measure supported on $\mathcal{C}$ is zero.

(b) Any homoclinic class $H_{f}(p)$ has a partially hyperbolic structure

$$
T_{H_{f}(p)} M=E^{s} \oplus E_{1}^{c} \oplus \cdots \oplus E_{k}^{c} \oplus E^{u} .
$$

Moreover, the minimal stable dimension of the periodic orbits of $H_{f}(p)$ is $\operatorname{dim} E^{s}$ or $\operatorname{dim} E^{s}+1$. Similarly, the maximal stable dimension of the periodic orbits of $H_{f}(p)$ is $\operatorname{dim} E^{s}+k$ or $\operatorname{dim} E^{s}+k-1$. For every $i=1, \ldots, k$, there exist periodic points in $H_{f}(p)$ whose Lyapunov exponent along $E_{i}^{c}$ is arbitrarily close to 0 . In particular, if $f \in \mathcal{G}$, then $f$ is partially hyperbolic.

Recently, Pacifico and Vieitez [20] proved that there is a residual subset $\mathcal{G}$ of $\operatorname{Diff}(M) \backslash \overline{\mathcal{H T}}$ such that for any Borel probability measure $\mu$ absolutely continuous with respect to Lebesgue, $f$ is $\mu$-expansive. Lee [15] showed that there is a partially hyperbolic diffeomorphism such that it is not measure expansive. From the facts, we consider continuum-wise expansive for partially hyperbolic diffeomorphisms. The set $\lambda$ is transitive if there is a point $x \in \Lambda$ such that $\omega(x)=\Lambda$, where $\omega(x)$ the omega limit set of $f$. we say that the set $\Lambda$ is robustly transitive if there are a $C^{1}$-neighborhood $\mathcal{U}(f)$ of $f$ and a neighborhood $U$ of $\Lambda$ such that for any $g \in \mathcal{U}(f), \Lambda_{g}(U)=\bigcap_{n \in \mathbb{Z}} g^{n}(U)$ is transitive, where $\Lambda_{g}(U)$ is the continuation of $\Lambda$. Let $M=\mathbb{T}^{3}$ and let $f: \mathbb{T}^{3} \rightarrow \mathbb{T}^{3}$ be a diffeomorphism.

Theorem B There is a $C^{1}$ neighborhood $\mathcal{U}(f)$ of $f \in \mathcal{R} \mathcal{T}\left(\mathbb{T}^{3}\right)$ and a residual set $\mathcal{R} \subset \mathcal{U}(f)$ such that for any $g \in \mathcal{R}, g$ is not continuum-wise expansive, where $\mathcal{R} \mathcal{T}\left(\mathbb{T}^{3}\right)$ is the set of all robustly transitive diffeomorphisms on $\mathbb{T}^{3}$.

\section{Proof of Theorems}

\subsection{Proof of Theorem A}

Let $M$ be as before, and let $f \in \operatorname{Diff}(M)$. The following Franks' lemma [9] will play essential roles in our proofs.

Lemma 2.1 Let $\mathcal{U}(f)$ be any given $C^{1}$ neighborhood of $f$. Then there exist $\epsilon>0$ and a $C^{1}$ neighborhood $\mathcal{U}_{0}(f) \subset \mathcal{U}(f)$ of $f$ such that for given $g \in \mathcal{U}_{0}(f)$, a finite 
set $\left\{x_{1}, x_{2}, \cdots, x_{N}\right\}$, a neighborhood $U$ of $\left\{x_{1}, x_{2}, \cdots, x_{N}\right\}$ and linear maps $L_{i}$ : $T_{x_{i}} M \rightarrow T_{g\left(x_{i}\right)} M$ satisfying $\left\|L_{i}-D_{x_{i}} g\right\| \leq \epsilon$ for all $1 \leq i \leq N$, there exists $\widehat{g} \in \mathcal{U}(f)$ such that $\widehat{g}(x)=g(x)$ if $x \in\left\{x_{1}, x_{2}, \cdots, x_{N}\right\} \cup(M \backslash U)$ and $D_{x_{i}} \widehat{g}=L_{i}$ for all $1 \leq i \leq N$.

The following was proved by [15]. For convenience, we give the proof in the section.

Lemma 2.2 If $f \in \operatorname{Diff}(M)$ has a non-hyperbolic periodic point, then for any neighborhood $\mathcal{U}(f)$ of $f$ and any $\eta>0$, there are $g \in \mathcal{U}(f)$ and a curve $\gamma$ with the following property:

1. $\gamma$ is $g$ periodic, that is, there is $n \in \mathbb{Z}$ such that $g^{n}(\gamma)=\gamma$;

2. the length of $g^{i}(\gamma)$ is less than $\eta$, for all $i \in \mathbb{Z}$;

3. $\gamma$ is normally hyperbolic with respect to $\mathrm{g}$.

Proof. Let $\mathcal{U}(f)$ be a $C^{1}$ neighborhood of $f$. Suppose $p$ is not hyperbolic periodic point of $f$. For simplicity, we may assume that $p$ is a fixed point of $f$. By Lemma 2.1, there is $g \in \mathcal{U}(f)$ such that $D_{p} g^{\pi(p)}$ has an eigenvalue $\lambda$ with $|\lambda|=1$. Then $g(p)=p_{g}$ and $T_{p_{g}} M=E_{p_{g}}^{s} \oplus E_{p_{g}}^{c} \oplus E_{p_{g}}^{u}$, where $E_{p_{g}}^{s}$ is the eigenspace corresponding to the eigenvalues with modulus less than $1, E_{p_{g}}^{u}$ is the eigenspace corresponding to the eigenvalues with modulus more than 1 , and $E_{p_{g}}^{c}$ is the eigenspace corresponding to $\lambda$. If $\lambda \in \mathbb{R}$ then $\operatorname{dim} E_{p_{g}}^{c}=1$ and if $\lambda \in \mathbb{C}$ then $\operatorname{dim} E_{p}^{c}=2$.

In the proof, we consider $\operatorname{dim} E_{p_{g}}^{c}=1$. For case $\operatorname{dim} E_{p_{g}}^{c}=2$, we can obtain the result as in the case $\operatorname{dim} E_{p_{g}}^{c}=1$.

Since $\operatorname{dim} E_{p_{g}}^{c}=1$ we assume that $\lambda=1$. By Lemma 2.1, there are $\epsilon>0$ and $h \in \mathcal{U}(f)$ such that

- $h\left(p_{g}\right)=g\left(p_{g}\right)=p_{g}$,

- $h(x)=\exp _{p_{g}} \circ D_{p_{g}} g \circ \exp _{p_{g}}^{-1}(x)$ if $x \in B_{\epsilon}\left(p_{g}\right)$, and

- $h(x)=g(x)$ if $x \notin B_{4 \epsilon}\left(p_{g}\right)$.

Since $\lambda=1$, we can construct a closed small arc $\mathcal{I}_{p_{g}} \subset B_{\epsilon}\left(p_{g}\right) \cap \exp _{p_{g}}\left(E_{p_{g}}^{c}(\epsilon)\right)$ with its center at $p_{g}$ such that

$\cdot \operatorname{diam} \mathcal{I}_{p_{g}}=\epsilon / 4$

$\cdot h\left(\mathcal{I}_{p_{g}}\right)=\mathcal{I}_{p_{g}}$, and

- $\left.h\right|_{\mathcal{I}_{p g}}$ is the identity map. 
Here $E_{p_{g}}^{c}(\epsilon)$ is the $\epsilon$-ball in $E_{p_{g}}^{c}$ centered at the origin $\vec{o}_{p}$. Then $\mathcal{I}_{p_{g}}$ is normally hyperbolic with respect to $h$, and for any $\eta<\epsilon / 4$, the length of $\mathcal{I}_{p_{g}}$ is less than $\eta$.

By the persistency of normally hyperbolic, we know that there is a neighborhood $\mathcal{U}(g)$ of $g$ such that for any $\tilde{g} \in \mathcal{U}(g)$ there is a curve $\tilde{\gamma}$ close to $\gamma$ such that all properties of $\gamma$ listed in the Lemma 2.2 is also satisfied for $\tilde{\gamma}$.

For $f \in \operatorname{Diff}(M)$, we say that $f$ is the star diffeomorphism (or $f$ satisfies the star condition) if there is a $C^{1}$-neighborhood $\mathcal{U}(f)$ of $f$ such that all periodic points of $g \in \mathcal{U}(f)$ are hyperbolic. Denote by $\mathcal{F}(M)$ the set of all star diffeomorphisms. Aoki [2] and Hayashi [11] showed that for any dimension case, if $f \in \mathcal{F}(M)$ then $f$ is Axiom A without cycles.

Lemma 2.3 [15, Lemma 3.4] There is a residual set $\mathcal{G} \subset \operatorname{Diff}(M)$ such that for any $f \in \mathcal{G}$,

- either $f$ is a star,

- or for any $\varepsilon>0$ there is a periodic curve $\gamma$ such that the length of $f^{n}(\gamma)$ is less than $\varepsilon$, for any $n \in \mathbb{Z}$.

Proof. Let $\mathcal{H}_{n}$ be the set of $C^{1}$ diffeomorphisms $f$ such that $f$ has a normally hyperbolic $\gamma$ which is $1 / n$-simply periodic curve. Since $\gamma$ is normally hyperbolic, we know that $\mathcal{H}_{n}$ is open. Let $\mathcal{N}_{n}=\operatorname{Diff}(M) \backslash \overline{\mathcal{H}_{n}}$. Then $\mathcal{H}_{n}(\eta) \cup \mathcal{N}_{n}(\eta)$ is open and dense in $\operatorname{Diff}(M)$. Let $\mathcal{G}=\bigcap_{n \in \mathbb{N}^{+}}\left(\mathcal{H}_{n} \cup \mathcal{N}_{n}\right)$. Then $\mathcal{G}$ is $C^{1}$ residual in $\operatorname{Diff}(M)$. Let $f \in \mathcal{G}$ and assume $f$ is not a star diffeomorphism, we know that $f \in \overline{\mathcal{H}_{n}}$ for any $n \in \mathbb{N}^{+}$by Lemma 2.2. Hence $f \notin \mathcal{N}_{n}$ and $f \in \mathcal{H}_{n}$ for any $n$. We know that $f$ has a normally hyperbolic $\gamma$ which is $\varepsilon$-simply periodic curve, for any $\varepsilon>0$.

The following was proved by [5, Lemma 2.2].

Lemma 2.4 Let $C \subset M$ be a continuum. $f$ is continuum-wise expansive if and only if there is $\delta>0$ such that for all $x \in M$, if a continuum $C \subset \Gamma_{\delta}(x)$ then $C$ is a singleton.

Proof of Theorem A. Let $f \in \mathcal{G}$ be continuum-wise expansive. Suppose by contradiction that $f \notin \mathcal{F}(M)$. From Lemma 2.3, for any $\varepsilon>0$ there is a periodic curve $\gamma$ such that the length of $f^{i}(\gamma)$ is less than $\varepsilon$, for any $i \in \mathbb{Z}$. Let $\Gamma_{\epsilon}(x)=\left\{x \in M: d\left(f^{i}(x), f^{i}(y)\right) \leq \epsilon\right.$ for all $\left.i \in \mathbb{Z}\right\}$. Since $f^{n}(\gamma)=\gamma$ for some $n \in \mathbb{Z}$, we know $\gamma \subset \Gamma_{\epsilon}(x)$. By Lemma 2.4, $\gamma$ should be a singleton which is a contradiction since $\gamma$ is a nontrivial continuum. Thus if $f \in \mathcal{G}$ is continuum-wise expansive then it is Axiom A without cycles. 


\subsection{Proof of Theorem B}

In this section, let $M=\mathbb{T}^{3}$ and let $f: \mathbb{T}^{3} \rightarrow \mathbb{T}^{3}$ be a diffeomorphism. In [17, Theorem B], Mañé constructed a robustly nonhyperbolic transitive diffeomorphism $f \in \operatorname{Diff}\left(\mathbb{T}^{3}\right)$. By [ $\left[\right.$, Theorem B], every robustly transitive diffeomorphism $f$ on $\mathbb{T}^{3}$ is partially hyperbolic. Thus we can find a partially hyperbolic diffeomorphism $f$ on $\mathbb{T}^{3}$ such that $f$ is robustly nonhyperbolic transitive.

Remark 2.5 There is a partially hyperbolic diffeomorphism $f$ on $\mathbb{T}^{3}$ such that $f$ is robustly nonhyperbolic transitive.

Lemma 2.6 [8, Corollary D] and [1, Theorem 4.10] There is a residual set $\mathcal{G}_{1} \subset$ $\mathcal{R} \mathcal{T}\left(\mathbb{T}^{3}\right)$ such that for any $f \in \mathcal{G}_{1}, f$ is strongly partially hyperbolic, and $\mathbb{T}^{3}$ is the homoclinic class $H_{f}(p)$, for some hyperbolic periodic point $p$.

Let $M$ be a closed smooth $n(\geq 2)$-dimensional manifold, and let $f: M \rightarrow M$ be a diffeomorphism. The following notion was introduced by Yang and Gan [24]. For any $\epsilon>0$, a $C^{1}$ curve $\eta$ is called a $\epsilon$-simply periodic curve of $f$ if

(i) $\eta$ is diffeomorphic to $[0,1]$ and its two endpoints are hyperbolic periodic points of $f$,

(ii) $\eta$ is periodic with period $\pi(\eta)$ and $L\left(f^{i}(\eta)\right)<\epsilon$ for any $i \in\{1,2, \cdots, \pi(\eta)\}$, where $L(\eta)$ denotes the length of $\eta$, and

(iii) $\eta$ is normally hyperbolic.

Lemma 2.7 [24, Lemma 2.1] There is a residual set $\mathcal{G}_{2} \subset \operatorname{Diff}(M)$ such that for any $f \in \mathcal{G}_{2}$, and any hyperbolic periodic point $p$ of $f$, we have the following:

for any $\epsilon>0$, if for any $C^{1}$ neighborhood $\mathcal{U}(f)$ of $f$ some $g \in \mathcal{U}(f)$ has a $\epsilon$ simply periodic curve $\eta$ such that two endpoints of $\eta$ are homoclinically related with $p_{g}$ then $f$ has an $2 \epsilon$-simply periodic curve $\zeta$ such that the two endpoints of $\zeta$ are homoclinically related to $p$.

Proof of Theorem B. Let $\mathcal{U}(f)$ be a $C^{1}$ neighborhood of $f \in \mathcal{R} \mathcal{T}\left(\mathbb{T}^{3}\right)$ and let $f \in$ $\mathcal{G}=\mathcal{G}_{1} \cap \mathcal{G}_{2}$. Let $p$ be a hyperbolic periodic point of $f$. Then for any $g \in \mathcal{G} \cap \mathcal{U}(f)$, we have $\mathbb{T}^{3}=H_{g}\left(p_{g}\right)$, where $p_{g}$ is the continuation of $p$. Since $H_{g}\left(p_{g}\right)$ is not hyperbolic, from [23, section 4], for any $\epsilon>0$, there is $g_{1} \in \mathcal{G} \cap \mathcal{U}(f)$ such that $g_{1}$ has an

$\epsilon$-simply periodic curve $\eta$, whose endpoints are homoclinically related to $p_{g_{1}}$. Note that $\eta$ is a closed and connected set, so it is a nontrivial continuum. Let $e=2 \epsilon$ be 
an expansive constant of $g_{1}$. For $x \in \mathbb{T}^{3}$, we set $\Gamma_{e}(x)=\left\{y \in \mathbb{T}^{3}: d\left(g_{1}^{i}(x), g_{1}^{i}(y)\right) \leq e\right.$ for all $i \in \mathbb{Z}$ \}. Since $g_{1}^{\pi\left(p_{g}\right)}(\eta)=\eta$, we have

$$
L\left(g_{1}^{i \pi\left(p_{g}\right)}(\eta)\right)=L(\eta)<e .
$$

Clearly $\eta \subset \Gamma_{e}(x)$. Since $\eta$ is compact and connected, and so, $\eta$ is not singleton which is a contradiction.

\section{Acknowledgement}

This work is supported by Basic Science Research Program through the National Research Foundation of Korea (NRF) funded by the Ministry of Science, ICT \& Future Planning (No-2014R1A1A1A05002124).

\section{References}

[1] F. Abdenur, C. Bonatti and S. Crovisier, Nonuniform hyperbolicity for $C^{1}$ generic diffeomorphisms, Isral J. Math., 183(2011), 1-60.

[2] N. Aoki, The set of Axiom A diffeomorphisms with no cycles. Bol. Soc. Bras. Mat., 23(1992), 21-65.

[3] A. Arbieto, Periodic orbits and expansiveness, Math. Z., 269(2011), 801-807.

[4] A. Artigue, Robustly N-expansive surface diffeomorphisms, Disc. Contin. Dynam. Syst. 36 (2016), 2367-2376.

[5] A. Artigue and D. Carrasco-Olivera, A note on measure expansive diffeomorphisms, J. Math. Anal. Appl., 428(2015), 713-716.

[6] K. Burns and A. Wilkinson, On the ergodicity of partilly hyperbolic systems, Ann. of Math. 171(2010), 451-489.

[7] S. Crovisier, M. Sambarino and D. Yang, Partial hyperbolicity and homoclinic tangencies, J. Eur. Math. Soc, 17(2015), 1-49.

[8] L. J. Díaz, E. R. Pujals and R. Ures, Partial hyperbolicity and robust transitivity, Acta Math., 183(1999), 1-43.

[9] J. Franks, Necessary conditions for stability of diffeomorphisms, Trans. Amer. Math. Soc. 158 (1971), 301-308. 
[10] J. Franks and C. Robinson, A quasi-Anosov diffeomorphism that is not Anosov Trans. Amer. Math. Soc, 223 (1976), 267-278.

[11] S. Hayashi, Diffeomorphisms in $\mathcal{F}^{1}(M)$ satisfy Axiom A, Ergodic Thoery \& Dynam. Syst., 12 (1992), 233-253.

[12] H. Kato, Continuum-wise expansive homeomorphisms, Can. J. Math., 45(1993), 576-598.

[13] M. Lee, General Expansiveness for Diffeomorphisms from the Robust and Generic Properties, to appear in J. Dynam. Cont. Syst.

[14] M. Lee, Continuum-wise expansive homoclinic classes for generic diffeomorphisms, Publ. Math. Debrecen 88(2016), 193-200.

[15] M. Lee, Measure expansiveness for generic diffeomorphisms, preprint.

[16] R. Mañé, Expansive diffeomorphisms, Lecture Notes in Math. 468, Springer, Berlin 1975.

[17] R. Mañé, Contributions to the stability conjecture, Topology, 17(1978), 383-396.

[18] C. A. Morales, A generalization of expansivity, Disc. Contin. Dynam. Syst. 32 (2012), 293-301.

[19] C. A. Morales and V. F. Sirvent, Expansive measures, 29 Colóquio Brasileiro de Matemática, 2013.

[20] M. J. Pacifico and J. L. Vieites, On measure expansive diffeomorphisms, Proc. Amer. Math. Soc., 143(2015), 811-819.

[21] K. Sakai, Continuum-wise expansive diffeomorphisms, Publca. Mate. 41(1997), 375-382.

[22] K. Sakai, N. Sumi, and K. Yamamoto, Measure-expansive diffeomorphisms, J. Math. Anal. Appl. 414 (2014), 546.552.

[23] M. Sambarino and J. Vieitez, On $C^{1}$-persistently expansive homoclinic classes, Discrete Contin. Dynam. Syst., 14(2006), 465-481.

[24] D. Yang and S. Gan, Expansive homoclinic classes, Nonlinearity, 22(2009), 729-733. 